\title{
Tailored treatment for the management of scleral necrosis following pterygium excision
}

\author{
LINNA LU*, SHIQIONG XU*, SHENGFANG GE, CHUNYI SHAO, ZI WANG, \\ XUYANG WENG, WENJUAN LU, XINHUA WU, YAO FU and XIANQUN FAN \\ Department of Ophthalmology, Ninth People's Hospital, \\ Shanghai Jiaotong University School of Medicine, Shanghai 200011, P.R. China
}

Received July 27, 2015; Accepted September 13, 2016

DOI: $10.3892 /$ etm.2017.4038

\begin{abstract}
The present study aimed to investigate the efficacy of tailored treatment for the management of scleral necrosis following pterygium surgery. A series of nine cases of scleral necrosis following pterygium excision between September 2009 and September 2012 were included. In cases where ischemia was the cause of scleral necrosis, Tenon's membrane covering (TMC) surgery was performed. For cases with surgically-induced necrotizing scleritis (SINS), systemic immunosuppressive therapy following surgical repair of the scleral defect was administered in the form of oral prednisolone (starting dose, 30-60 mg/day). Five patients with ischemic scleral necrosis received TMC postoperatively. Four patients with SINS received various doses of oral prednisolone according to their systematic immune state. All patients had successful postoperative results except one with rheumatoid arthritis, who postoperatively developed scleral patch graft melting within 2 weeks. Following aggressive immunosuppressive treatment, the scleral patch graft was saved. In conclusion, patients achieved positive results using tailored treatment according to the pathogenesis of their scleral necrosis.
\end{abstract}

Correspondence to: Professor Yao Fu or Professor Xianqun Fan, Department of Ophthalmology, Ninth People's Hospital, Shanghai Jiaotong University School of Medicine, 639 Zhizaoju Road, Shanghai 200011, P.R. China

E-mail: fuyaofy@sina.com

E-mail: fanxq@sh163.net

*Contributed equally

Abbreviations: CA, conjunctival autograft; MMC, mitomycin C; ESR, erythrocyte sedimentation rate; CRP, C-reactive protein; NSAID, nonsteroidal anti-inflammatory drug; TMC, Tenon's membrane covering; AMT, amniotic membrane transplantation; CPG, corneoscleral patch graft; SINS, surgically induced necrotizing scleritis

Key words: scleral necrosis, pterygium, Tenon's membrane, systemic immunosuppressive therapy

\section{Introduction}

Pterygium surgery has changed over the past decade, and several techniques are currently available to the ophthalmic surgeon. Conjunctival autograft (CA) and CA combined with adjunctive mitomycin $\mathrm{C}$ (MMC) are commonly performed procedures for the treatment of primary and recurrent pterygium (1-3).

Scleral necrosis/melting is a rare complication that may develop following pterygium surgery. The possible pathological mechanisms underlying scleral necrosis following pterygium surgery include infection, hypersensitivity response and ischemia; the latter may serve an important role, and the use of adjunctive MMC may increase its likelihood (4).

Treatments include the use of antibiotics, systemic immunosuppressive drugs and surgical repair of the scleral defect with a variety of graft materials. In a number of studies, high doses of systemic immunosuppressive therapy are described as being necessary for the arrest of the progression of the melting process (4-7), although they have a number of side effects.

In the current study, personalized treatment tailored to the pathogenesis was used as an alternative to high doses of systemic immunosuppressive therapy. The present study aimed to investigate the efficacy of tailored treatment for the management of scleral necrosis following pterygium surgery. Herein, nine cases of scleral necrosis following pterygium excision are presented and their pathogenesis and management are discussed.

\section{Subjects and methods}

Study design. This retrospective study was performed according to the principles of the Declaration of Helsinki, informed consent was obtained from the patients, and the Ninth People's Hospital, Shanghai JiaoTong University School of Medicine Ethics Committee (Shanghai, China) approved the study. A series of nine cases of scleral necrosis following pterygium excision between September 2009 and September 2012 were evaluated retrospectively. The patients had undergone nasal or temporal pterygium excision in a number of local hospitals, and were recommended to the Department of Ophthalmology, Ninth People's Hospital, by their primary physicians. 
Participants. All the individuals included in the present study underwent a comprehensive ophthalmologic examination and routine diagnostic evaluation; initial treatment and the degree of scleral necrosis were recorded.

Laboratory evaluations were performed to search for an inflamed, autoimmune or infectious etiology of the necrotizing scleritis. These laboratory evaluations included the following: Scrapes from the surface of the graft sent for smear and culture examination; routine blood [blood cell count, erythrocyte sedimentation rate (ESR), C-reactive protein [CRP] expression level and serum uric acid estimation]; extensive blood tests for autoimmune diseases (rheumatoid factor, antinuclear antibodies, anti-DNA antibodies, antinuclear antigen antibodies, and complement and thyroid antibodies); serology (human immunodeficiency virus 1 and 2, varicella-zoster virus, hepatitis B virus, hepatitis C virus, Venereal Disease Research Laboratory test and Treponema pallidum haemagglutination assay); chest radiography; and Mantoux test for tuberculosis.

Scleral melt severity was categorized as follows: A, scleral thinning without corneal melting; B, scleral thinning with corneal melting; and $\mathrm{C}$, scleral thinning with uveal exposure.

Treatments were based on ophthalmic examination, medical history of the patients and laboratory results.

Surgical technique. The surgery was performed under peribulbar anesthesia [0.75\% bupivacaine (Sensorcaine; Harvest Pharmaceutical Co., Ltd.); $2 \%$ lidocaine (Xylocaine; Harvest Pharmaceutical Co., Ltd.)]. Surgical techniques were chosen on the basis of the degree of scleral melting, and included Tenon's membrane covering (TMC) combined with amniotic membrane transplantation (AMT) and a corneoscleral patch graft (CPG) combined with AMT.

For TMC, the conjunctiva, Tenon's membrane and episcleral tissue were dissected carefully to expose the defect in the sclera. All devitalized, infected and necrotic soft tissue was debrided and the adjacent unaffected tissue was preserved. Subsequently, Tenon's membrane was dissected around the lesion area and extended along the surface of the globe to cover the lesion completely, followed by closure. If the adjacent Tenon's membrane could not be mobilized, a rotational Tenon's membrane autograft was used. Finally, the area was covered with an amniotic membrane (Fig. 1).

For CPG, the donor sclera was soaked three times in Ringer's lactate solution (Minsheng Pharmaceutical Group Co., Ltd., Hangzhou, China) for $10 \mathrm{~min}$, in betadine (Yongan Pharmaceutical Group Co., Ltd., Chengdu, China) for $10 \mathrm{~min}$ and finally in $20 \mathrm{mg} / \mathrm{ml}$ gentamicin solution (Yaoyou Pharmaceutical Group Co., Ltd., Chongqing, China) for $10 \mathrm{~min}$, followed by shaping to fit the defect area after all devitalized and necrotic tissue had been debrided. The graft was then secured to the edges of the resection site using 8-0 Vicryl sutures on the scleral side and 10-0 nylon sutures on the corneal side (if required). The repaired sclera was covered with an amniotic membrane graft.

For AMT, following TMC or CPG, the corneoscleral patch or Tenon's membrane patch was covered with amniotic membrane, with the stromal side facing down; 10/0 monofilament nylon sutures were used to suture the surrounding conjunctiva.

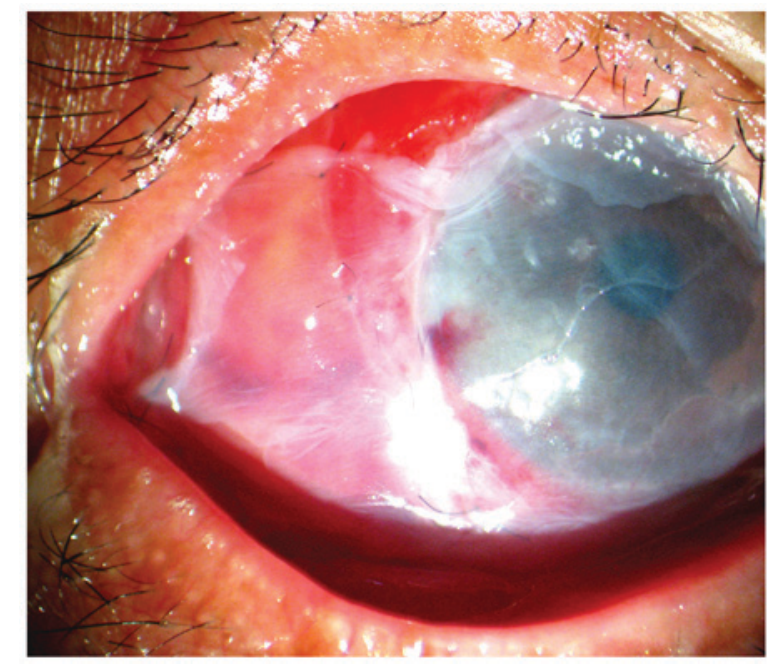

Figure 1. A defect area was covered with a Tenon's membrane flap from the surrounding site following removal of the devitalized tissue and surrounding inflamed conjunctiva. Then, the repaired sclera was covered with amniotic membrane.

Medication. Postoperatively, all patients were administered topical $0.1 \%$ dexamethasone (4 times daily; Alcon, Fort Worth, TX, USA), $0.3 \%$ ciprofloxacin (4 times daily; Wujing Pharmaceutical Co., Ltd., Wuhan, China) and artificial tears (6 times daily; Alcon) for 1 month. Systemic immunosuppressive therapy, oral prednisolone (30 mg/day for 2 weeks; Lijun Pharmaceutical Co., Ltd.), was administered prior to tapering of the dosage; however, if laboratory results confirmed the existence of immune dysfunction, prednisolone $(60 \mathrm{mg} / \mathrm{day})$ was used as the starting dose. Low-dose maintenance corticosteroid therapy (5 $\mathrm{mg} /$ day prednisolone) was required for 3 months. Antimicrobial treatment $(0.3 \%$ ciproflaxin) was administered according to the results of antimicrobial sensitivity testing.

\section{Results}

Patient clinical features. Nine patients (four male and five female) were included in this study. The right eye was affected in five patients, and the left eye was affected in four. Their age ranged between 41 and 71 years old, with an average age of 57 years.

Six patients had undergone uneventful pterygium surgery with conjunctival autografting (CA) combined with MMC (0.02\% MMC for $2 \mathrm{~min}$ ). Case nos. 6, 7 and 9 had undergone nasal pterygium excision with CA, but did not have adjunctive MMC intraoperatively. All patients had presented with severe pain and redness in the eye $\sim 2-8$ weeks postoperatively. In the local hospital, all patients had received administration of steroid and antibiotic eye drops, two patients had accepted oral nonsteroidal anti-inflammatory drugs, six had undergone AMT, and one was administered oral prednisone at $10 \mathrm{mg}$ for 5 days. However, all these methods were unable to arrest the progression of the necrotizing process. Three patients presented with degree A of scleral necrosis; the underlying sclera was necrotic, avascular and thinning, but with no evidence of active corneal melting (case nos. 2,4 and 5). Four patients presented with degree $\mathrm{B}$ of scleral necrosis (case 

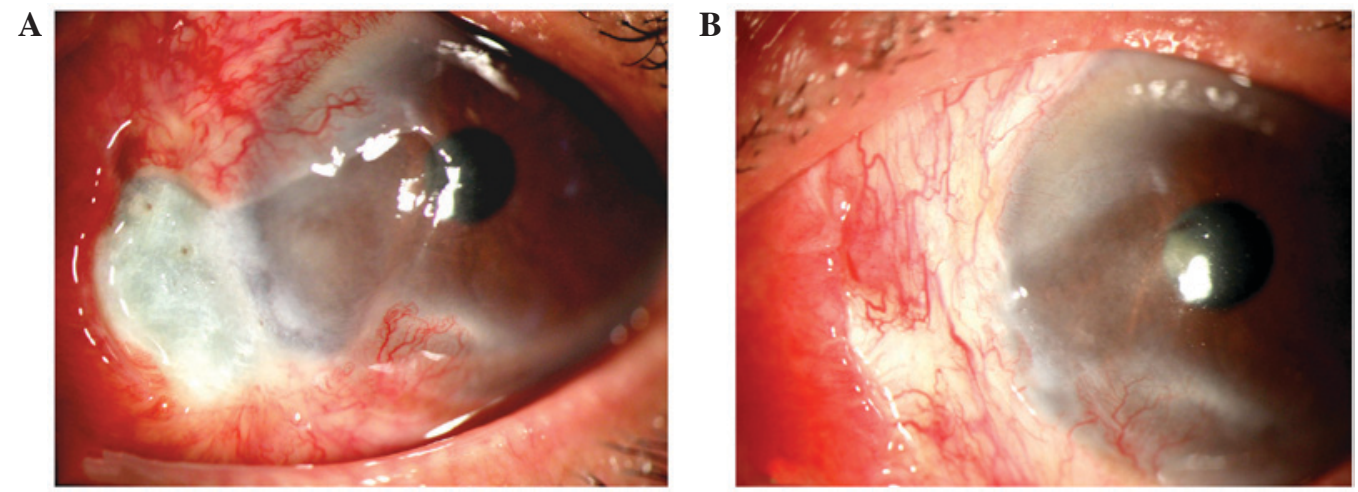

Figure 2. (A) A patient presented the moderate scleral thinning associated with corneal melting. (B) At the one month follow-up, the patient's scleral necrosis disappeared progressively accompanied with normalization of the sclera, and conjunctival and corneal reepithelialization.
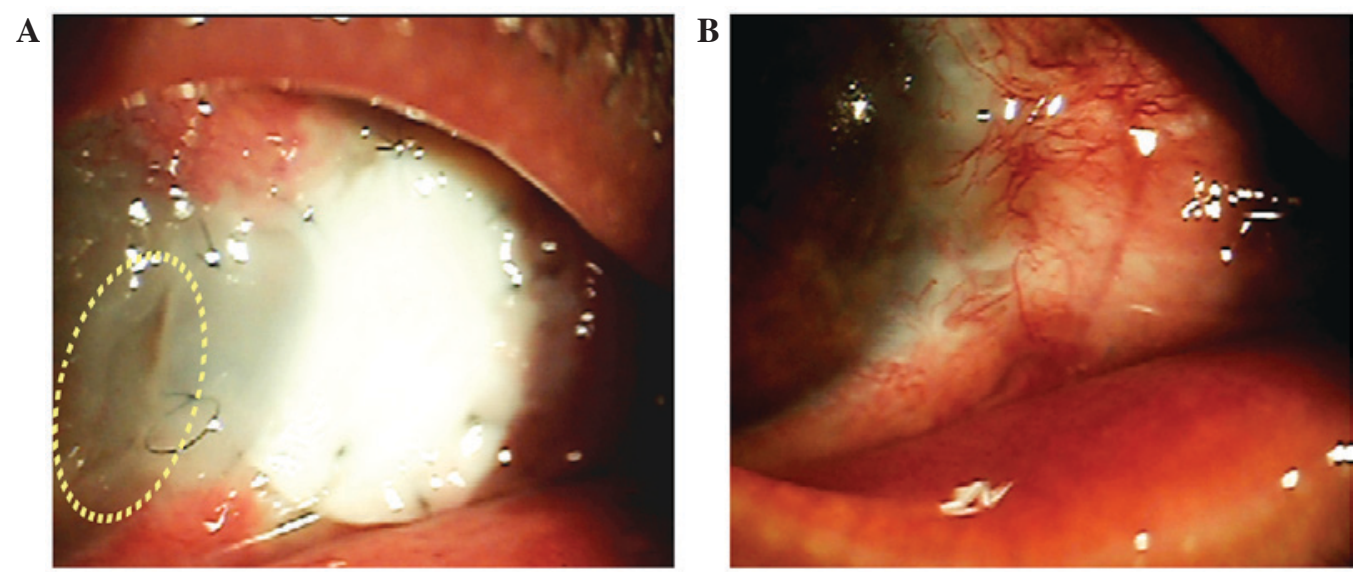

Figure 3. (A) A patient with rheumatoid arthritis, $\sim 2$ weeks after surgery, whose corneal margin of the graft began to melt (highlighted by the yellow circle) and the remainder of the graft appeared softened but intact over the defect without uveal exposure. (B) The area of scleral necrosis resolved after 8 weeks and the perforated area was sealed with vascularized epithelialization.

nos. 1,3,6 and 7) and two presented with degree $\mathrm{C}$ scleral necrosis (case nos. 8 and 9).

Four patients with negative past medical histories denied fever, night sweats, weight loss, malaise, joint pains, skin rashes and headaches. Three patients had hypertension. The remaining two patients had a significant past medical history; one was a 52-year-old male who had undergone repeated chemotherapy and radiotherapy because of lung cancer recurrence (case no. 8), and the other was a 54-year-old female with a medical history of rheumatoid arthritis (case no. 9).

Laboratory evaluation. Laboratory evaluation revealed that case no. 9 had a high CRP level (CRP, 24 mg/l; normal levels, $<10 \mathrm{mg} / \mathrm{l}$ ), an ESR of $90 \mathrm{~mm} / \mathrm{h}$ (normal levels, $<20 \mathrm{~mm} / \mathrm{h}$ in females or $<15 \mathrm{~mm} / \mathrm{h}$ in males), rheumatoid factor $(+)$ and anti-nuclear antigen antibodies (+). In addition, case no. 7 had a high ESR $(65 \mathrm{~mm} / \mathrm{h})$. Microbiologic studies for case no. 2 revealed the presence of Staphylococcus epidermidis, which is typically a saprophyte of the conjunctiva; in vitro antimicrobial testing showed that this strain was multiresistant but was sensitive to ciprofloxacin. The other patients showed no laboratory abnormalities, and the the microbiological investigations of case nos. 6, 7 and 9 were negative. These parameters were tabulated, summarized and analyzed accordingly (Table I).
Current intervention. Case nos. 1-7 received TMC combined with AMT; among them, case nos. 1-5 received no systemic immunosuppressive therapy, case no. 6 was administered oral prednisolone at $30 \mathrm{mg}$ /day for 2 weeks followed by tapering postoperatively, and case no. 7 was administered oral prednisolone $60 \mathrm{mg}$ /day because of his high ESR. For case nos. 8 and 9, because of the marked scleral thinning and uveal exposure, a CPG procedure was performed in combination with AMT. In addition, case no. 8 was administered oral prednisolone at $30 \mathrm{mg} /$ day for 2 weeks before tapering, and case no. 9 was administered $60 \mathrm{mg} /$ day for 2 weeks because of their history of rheumatoid arthritis and high CRP and ESR. Antibiotic treatment for case no. 2 comprised $0.3 \%$ ciprofloxacin 6 times daily.

Postoperative results. All patients experienced uncomplicated postoperative courses except case no. 9. In the patients, the scleral necrosis disappeared progressively within 3-8 weeks accompanied with normalization of the sclera, conjunctival reepithelialization and neoangiogenesis (Fig. 2). There were no relapses over a period of one-year follow-up.

In case no. 9, 2 weeks after surgery, the corneal margin of the graft started to melt and the remainder of the graft appeared softened but intact over the defect, without uveal exposure (Fig. 3A). At this stage, following rheumatology consultation, 


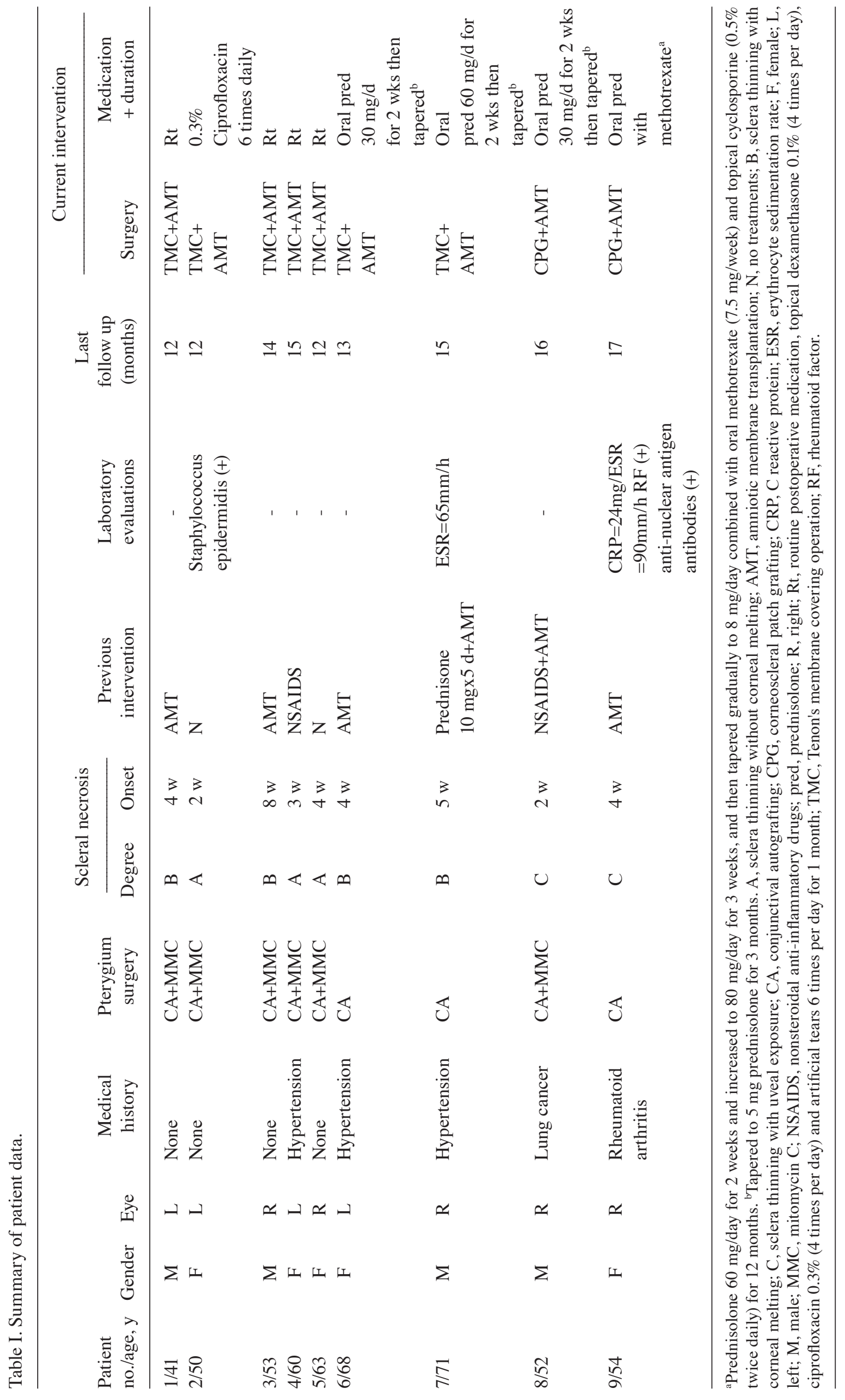


the prednisolone dose was increased to $80 \mathrm{mg} /$ day for 3 weeks, and then tapered gradually to the maintenance dose (8 mg/day) combined with oral methotrexate $(7.5 \mathrm{mg} /$ week; Xinyi, Shanghai, China) and topical cyclosporine (0.5\% twice daily; Novartis International AG, Basel, Switzerland) for 12 months. The area of scleral necrosis resolved after 8 weeks; meanwhile, the perforated area was sealed with vascularized epithelialization (Fig. 3B). The immunosuppressive drug doses remained unchanged during the one-year follow-up period, and no relapses occurred.

\section{Discussion}

Scleral necrosis is a rare but severe form of scleritis, which threatens the vision and integrity of the eye. It has been reported following all types of ocular surgery, but most commonly following pterygium excision $(4,5,8)$. Postoperative infection is one possible reason for the occurrence of scleritis following surgery. However, as with many reported cases described in the literature, smear examinations and cultures were negative in this study, except for case no. 2, whose microbiologic studies revealed the presence of Staphylococcus epidermidis which is typically a saprophyte of the conjunctiva. A hypersensitivity response to surgical trauma and scleral ischemia resulting from disruption of episcleral vasculature and manipulation of the previous surgical scar may be possible alternative explanations (9). Various methods have been proposed for the treatment of noninfectious scleral necrosis, but there is no consensus in the medical literature regarding its appropriate management. As described in the literature, besides reinforcement of the thinning or perforated sclera (9), high doses of systemic immunosuppressive therapy are typically recommended (7,10-12).

In the present study, case nos. 1-5 accepted the administration of $0.02 \% \mathrm{MMC}$ intraoperatively to prevent recurrence. $\mathrm{MMC}$ is an antineoplastic antibiotic alkylating agent that selectively inhibits DNA replication by forming covalent linkages with guanosine residues in DNA $(2,13)$; therefore, preventing proliferation of Tenon's fibroblasts (2). However, the delay in wound healing results in vascular compromise to the surgical site, and this, coupled with the permanent inhibition of fibroblast proliferation, exposes the sclera to avascular necrosis (14). This may be one explanation for the cases in the present study experiencing scleral necrosis. Excessive cauterization and conjunctival graft inversion during surgery are other possible causes.

As described above, ischemia is likely to be responsible for the occurrence of scleral necrosis. Therefore, if provision of vascular tissue to cover the defect can optimize the conditions for repair of the sclera, and allow the maintenance of blood flow to the grafted area, then relatively unaggressive therapy may be sufficient. Two options have been proposed to deal with this situation; the tarsoconjunctival pedicle flap (12) and conjunctiva-Müller muscle pedicle flap (15), but these methods are complicated and may cause problems such as ptosis, retraction of the eyelid, fornix shortening and eyelid margin deformities. According to the basic surgical principle of 'simpler is better', a Tenon's membrane flap is preferable to cover the lesion before the conventional procedure of scleral repair. The loose connective tissue of Tenon's capsule is highly vascularized; these vessels show a specialized morphology characterized by numerous arteriovenous anastomoses, and a muscle-rich venous network. Good ductility and good blood supply mean that the Tenon's membrane is suitable for repair of defects. In the present study, without any systemic immunosuppressive therapy, case nos. 1-5 had good postoperative courses, which confirms the feasibility of this method.

Reinforcement of the sclera is important, particularly when the uveal tract is exposed, to prevent prolapse of ocular contents and secondary infection. Amniotic membrane grafts and corneoscleral patch grafts are tissues commonly used for scleral reconstruction. In the present study, seven patients received TMC combined with AMT, but two patients with a risk of uveal exposure received CPG combined with AMT. Use of the amniotic membrane has a number of advantages, such as its easy availability, nonantigenic nature and lack of risk of immunologic rejection, which makes it an excellent graft and a substrate for ocular surface reconstruction (16). In addition, amniotic membrane contains many growth factors, promotes reepithelialization, and reduces fibrosis and inflammation, which benefit epithelialization. Covering the corneoscleral patch graft or Tenon's membrane with AMT is important, because it may help the ocular surface to reepithelialize rapidly to achieve a viable graft.

It is noteworthy that three of the patients in the present study developed scleral necrosis without the application of adjunctive MMC or intraoperative irradiation, and microbiological investigations were negative. Given the absence of these potential causes, the diagnosis of surgically induced necrotizing scleritis (SINS) was applied. Classical SINS is thought to be a delayed-type hypersensitivity response whereby mild surgical trauma or ischemia exposes tissue antigens, to which the immune system becomes sensitized $(5,17,18)$. Such hypotheses are supported by the success of systemic immunosuppressive therapy $(5,17,18)$, the presence of immune complexes in episcleral vessel walls $(17,19)$ and the high prevalence of vasculitis among such patients $(4,18,20-22)$.

In the present study, laboratory evaluation showed that case no. 9 had a high level of CRP and high ESR, and was positive for rheumatoid factor and antinuclear antigen antibodies. Case no. 7 had high ESR, suggesting the presence of immune dysfunction. In case no. 6, although there were no obvious abnormalities identified in extensive blood tests for autoimmune diseases, SINS was suspected because of the absence of the common pathogenetic mechanisms described above. For these patients with suspected SINS, systemic immunosuppressive therapy was administered based on the severity of the illness. Case no. 6 was administered oral prednisolone at $30 \mathrm{mg} /$ day for 2 weeks before the symptoms and signs allowed postoperative tapering, and cases no. 7 and 9 were administered prednisolone at $60 \mathrm{mg} /$ day for 2 weeks. In case nos. 6 and 7, besides a hypersensitivity reaction, the necrosis was attributable to the fragile vasculature attributable to their age and the presence of hypertension; therefore, TMC was applied to improve the blood supply. Although case no. 8 had received MMC previously, his rapid and severe disease progression suggested the presence of a hypersensitivity reaction, and therefore was administered prednisolone at $30 \mathrm{mg} /$ day. All the patients had uncomplicated postoperative courses except case no. 9. Case no. 9, a patient with rheumatoid 
arthritis, developed graft necrosis within 2 weeks following the scleral patch graft. In this particular case, the graft was covered with amniotic membrane that had retracted within 5 days, which may have delayed the epithelialization of the graft. Furthermore, the conjunctiva and Tenon's membrane were severely affected by the vasculitic process and they may not have provided sufficient vascular supply to the graft $(5,15)$. The current recommendation for this situation is the concomitant use of systemic immunosuppressive drugs, which reduces the severity of vasculitis and increases the chance of survival of the patch graft in such patients $(4,9)$. In this case, the high level of ESR and CRP and the melting graft postoperatively indicated the presence of active scleritis. Following consultation with a rheumatologist, more aggressive immunosuppressive therapy was adopted, which prevented the graft from melting.

Limitations of the present study included the relatively small number of patients and the retrospective data collection, primarily because of its infrequent collection following pterygium surgery. In addition, delayed type hypersensitivity and SINS are possible explanations for the cases described. Therefore, further observations and research concerning the underlying mechanisms of pathogenesis are required.

In conclusion, the possible pathogenetic mechanisms underlying scleral necrosis following surgery include infection, a hypersensitivity response and ischemia. By using different treatments tailored to the pathogenesis of a particular case, the present study was able to use relatively unaggressive therapy. For scleral necrosis caused by ischemia, Tenon's membrane flap may be a simple and safe treatment that avoids the need for high-dose immunosuppression, while for those with suspected SINS or infected cases, high-dose immunosuppression or appropriate antibiotics are necessary.

\section{Acknowledgements}

The present study was supported by the National Natural Science Foundation of China (grant nos. 81170876, 31271029 and 81320108010) and the Science and Technology Commission of Shanghai (grant nos. 13ZR1423600 and 12ZR1417300).

\section{References}

1. Kheirkhah A, Hashemi H, Adelpour M, Nikdel M, Rajabi MB and Behrouz MJ: Randomized trial of pterygium surgery with mitomycin $\mathrm{C}$ application using conjunctival autograft versus conjunctival-limbal autograft. Ophthalmology 119: 227-232, 2012.

2. Díaz L, Villegas VM, Emanuelli A and Izquierdo NJ: Efficacy and safety of intraoperative mitomycin $\mathrm{C}$ as adjunct therapy for pterygium surgery. Cornea 27: 1119-1121, 2008.
3. Fernandes M, Sangwan VS, Bansal AK, Gangopadhyay N Sridhar MS, Garg P, Aasuri MK, Nutheti R and Rao GN: Outcome of pterygium surgery: Analysis over 14 years. Eye (Lond) 19: 1182-1190, 2005.

4. Jain V, Shome D, Natarajan S and Narverkar R: Surgically induced necrotizing scleritis after pterygium surgery with conjunctival autograft. Cornea 27: 720-721, 2008.

5. Morley AM and Pavesio C: Surgically induced necrotising scleritis following three-port pars plana vitrectomy without scleral buckling: A series of three cases. Eye (Lond) 22: 162-164, 2008.

6. Sainz de la Maza M, Molina N, Gonzalez-Gonzalez LA, Doctor PP, Tauber J and Foster CS: Scleritis therapy. Ophthalmology 119: 51-58, 2012.

7. Young AL, Wong SM, Leung AT, Leung GY, Cheng LL and Lam DS: Successful treatment of surgically induced necrotizing scleritis with tacrolimus. Clin Experiment Ophthalmol 33: 98-99, 2005.

8. Yamazoe K, Shimazaki-Den S, Otaka I, Hotta K and Shimazaki J: Surgically induced necrotizing scleritis after primary pterygium surgery with conjunctival autograft. Clin Ophthalmol 5: 1609-1611, 2011.

9. Sangwan VS, Jain V and Gupta P: Structural and functional outcome of scleral patch graft. Eye (Lond) 21: 930-935, 2007.

10. Gregory ME, Weir CR and Ramaesh K: Excision of granulation tissue and free conjunctival autograft in the management of necrotizing scleritis. Cornea 29: 577-579, 2010.

11. Rachitskaya A, Mandelcorn ED and Albini TA: An update on the cause and treatment of scleritis. Curr Opin Ophthalmo 21: 463-467, 2010.

12. Davidson RS, Erlanger M, Taravella M, Gregory DG and Durairaj VD: Tarsoconjunctival pedicle flap for the management of a severe scleral melt. Cornea 26: 235-237, 2007.

13. Dadeya S and Fatima S: Comeoscleral perforation after pterygium excision and intraoperative mitomycin C. Ophthalmic Surg Lasers Imaging 34: 146-148, 2003.

14. Solomon A, Kaiserman I, Raiskup FD, Landau D and Frucht-Pery J: Long-term effects of mitomycin $\mathrm{C}$ in pterygium surgery on scleral thickness and the conjunctival epithelium. Ophthalmology 111: 1522-1527, 2004.

15. Yazici B: Use of conjunctiva-Müller muscle pedicle flap in surgical treatment of necrotizing scleritis. Ophthal Plast Reconstr Surg 24: 19-23, 2008.

16. Kim BH: Surgical treatment of necrotic scleral calcification using combined conjunctival autografting and an amniotic membrane inlay filling technique. Eye (Lond) 25: 1484-1490, 2011.

17. Díaz-Valle D, Benítez del Castillo JM, Castillo A, Sayagués O, Bañares A and García-Sánchez J: Immunologic and clinical evaluation of postsurgical necrotizing sclerocorneal ulceration. Cornea 17: 371-375, 1998

18. O'Donoghue E, Lightman S, Tuft S and Watson P: Surgically induced necrotising sclerokeratitis (SINS)-precipitating factors and response to treatment. Br J Ophthalmol 76: 17-21, 1992.

19. Fong LP, Sainz de la Maza M, Rice BA, Kupferman AE and Foster CS: Immunopathology of scleritis. Ophthalmology 98: 472-479, 1991.

20. Singh RP and McCluskey P: Scedosporium prolificans sclerokeratitis 10 years after pterygium excision with adjunctive mitomycin C. Clin Experiment Ophthalmol 33: 433-434, 2005.

21. Lai T, Leibovitch I, Zadeh R, Chehade M, Tamblyn D and Selva D: Surgically induced necrotizing scleritis occurring 48 years after strabismus surgery. J Pediatr Ophthalmol Strabismus 42: 180-182, 2005.

22. Akpek EK, Thorne JE, Qazi FA, Do DV and Jabs DA: Evaluation of patients with scleritis for systemic disease. Ophthalmology 111: 501-506, 2004. 